\title{
Kaon photoproduction and electroproduction near threshold*
}

\author{
T. Mart \\ Departemen Fisika, FMIPA, Universitas Indonesia, Depok 16424, Indonesia
}

(Dated: April 19, 2022)

\begin{abstract}
We analyze the electromagnetic production of $K^{+} \Lambda$ and $K^{0} \Lambda$ near their production thresholds by using isobar models. In the $K^{+} \Lambda$ channel we show that the model can nicely describe the available experimental data. In the $K^{0} \Lambda$ channel we demonstrate that the $K^{0}$ charge form factor has sizable effects on the longitudinal cross section. By extending the model up to $W=1730$ $\mathrm{MeV}$, we are able to observe the existence of the narrow $P_{11}\left(J^{p}=1 / 2^{+}\right)$resonance in the kaon photoproduction process. It is found that the most convincing mass (width) of this resonance is $1650 \mathrm{MeV}(5 \mathrm{MeV})$.

PACS numbers: 13.60.Le, 13.30.Eg, 25.20.Lj, 14.20.Gk
\end{abstract}

\footnotetext{
* Talk given at Baryons'10: International Conference on the Structure of Baryons, December 7-11, 2010, Osaka, Japan.
} 


\section{INTRODUCTION}

Threshold properties of meson photoproduction can provide invaluable information, since the production dynamics and the complexity level of the process can be substantially limited at threshold. Especially in the case of kaon photoproduction, for which the threshold energy is sufficiently large to allow for a number of nucleon resonances in the process. For energies currently available at nuclear physics accelerators, such as JLab in Newport News, Spring8 in Osaka, and MAMI in Mainz, around 15 nucleon resonance listed in the Particle Data Book

(PDG) [1] should be taken into account [2]. However, if we limit the energy of interest only up to $50 \mathrm{MeV}$ above the reaction threshold ( $W \approx 1660 \mathrm{MeV}$ ), only the $S_{11}(1650)$ resonance state may contribute to the process.

The $S_{11}(1650)$ is a four-star resonance. Since all of its properties are known, we can use the information from PDG to limit the number of uncertain free parameters. Therefore, the model can be constructed to explain experimental data more accurate than a global model that fits experimental data in a wide range of kinematics but tends to overlook small structures near threshold. An accurate model is inevitably required if we want to investigate the origin of these structures. Furthermore, since kaon electroproduction provides a unique way to study charged and neutral kaon form factors in the space-like region, an accurate phenomenological model is certainly well suited for this purpose.

Another interesting and relevant topic is the search for the nonstrange member of antidecuplet predicted by the chiral quark soliton model ( $\chi$ QSM) [3], which has a mass between 1650 and $1690 \mathrm{MeV}$, but very narrow width. In the case of $\pi N$ or $\eta N$ photoproduction, the corresponding c.m. energy is far from threshold. However, this is not the case for kaon photoproduction, e.g. $K^{+} \Lambda$, for which the threshold energy is $1610 \mathrm{MeV}$. Clearly, kaon photoproduction near threshold provides the best tool for this purpose.

\section{II. $K^{+} \Lambda$ PRODUCTION NEAR THRESHOLD}

Recently, we have investigated photoproduction of the $K^{+} \Lambda$ at energies near its production threshold by utilizing an isobar model [4]. The background amplitude of the model was obtained from a series of tree-level Feynman diagrams, whereas the resonance term for the $S_{11}(1650)$ state is calculated from the electric multipole $E_{0+}$. Although the number of free 

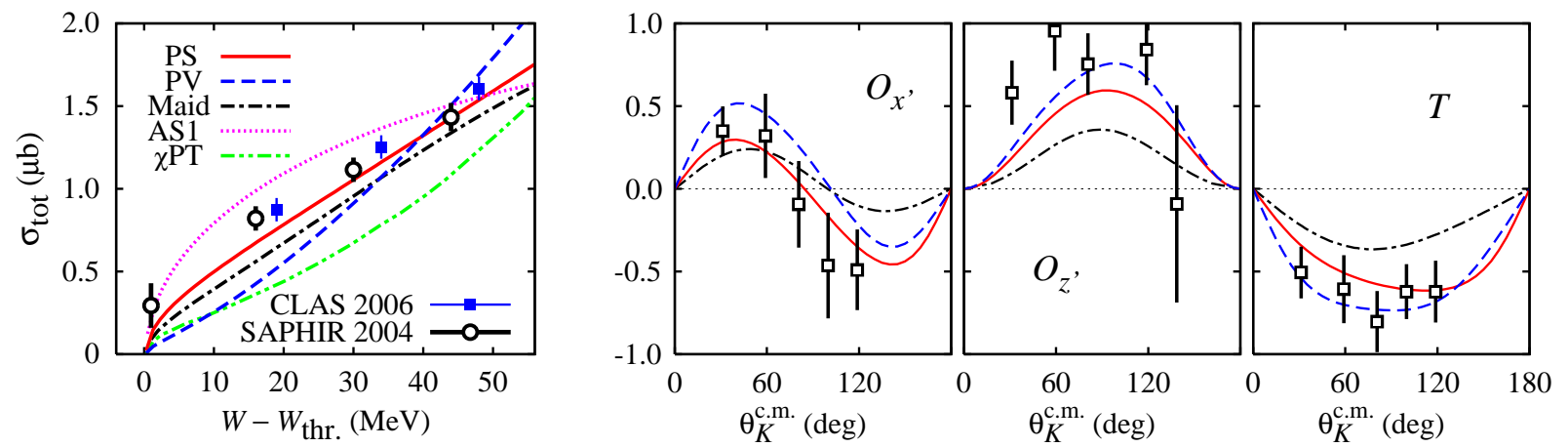

FIG. 1: (Left panel) Comparison between total cross sections calculated from the PS, PV, AS1 [5], and Kaon-Maid [6] models with the result of the chiral perturbation theory [7] and experimental data from the SAPHIR [8] and CLAS [9] collaborations. (Right panels) The beam-recoil double polarization observables $O_{x}, O_{z}$, and target asymmetry $T$ predicted by the PS, PV, and Kaon-Maid models [6] compared with experimental data from the GRAAL [10] collaboration. Notation of the curves is as in the left panel.

parameters is extremely reduced by exploiting the available information from PDG [1], the model can nicely describe experimental data both in the real and virtual photon sectors up to total c.m. energy $W=50 \mathrm{MeV}$ above the threshold.

It has been known that the pseudoscalar (PS) coupling can describe kaon photoproduction data better than the pseudovector (PV) coupling. This has been also found in our recent study, as shown in Fig. 1. The PV result underestimates the data up to $W=40 \mathrm{MeV}$ above threshold. The same deficiency is also shown by the Kaon-Maid model. As shown in the figure, prediction of chiral perturbation theory also underpredicts experimental data by about $30 \%-50 \%$. Interestingly, we observe that the prediction of the PV model is very close to the prediction of the chiral perturbation theory up to $W=10 \mathrm{MeV}$ above threshold. This fact originates from the small background terms of the PV model in this energy region. The AS1 model seems to overestimate most of the experimental data shown. Note that we did not use these total cross section data in our fits.

Comparison between experimental data and the predicted $O_{x}, O_{z}$ observables as well as the target asymmetry $T$ is shown in the three right panels of Fig. 11 Note that the calculated polarization observables shown here are pure prediction. Nevertheless, the predicted $O_{x}$ and $O_{z}$, as well as the target asymmetry $T$, are in perfect agreement with experimental data. 


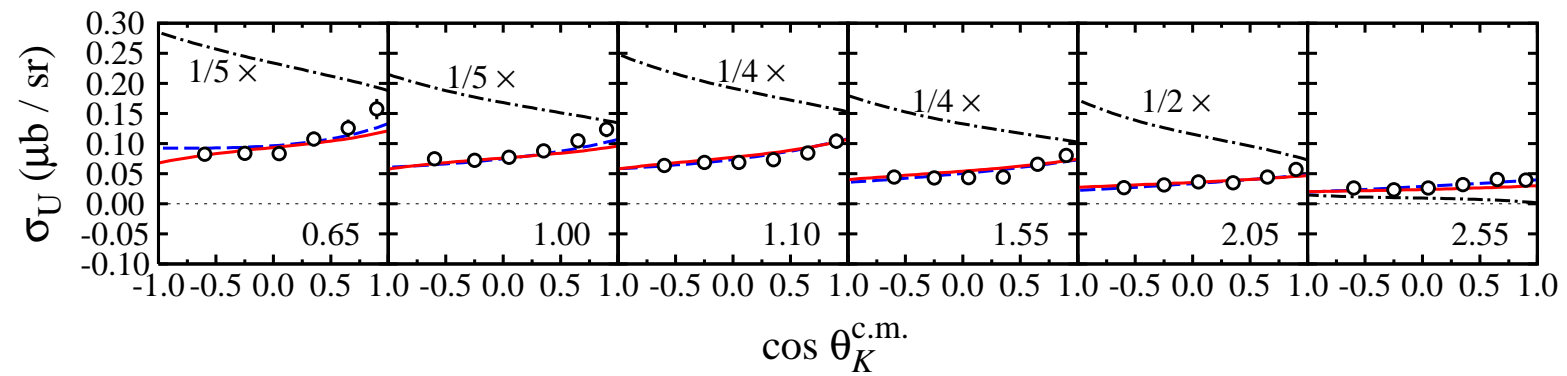

FIG. 2: Unpolarized differential cross sections for kaon electroproduction $e+p \rightarrow e^{\prime}+K^{+}+\Lambda$ as a function of the kaon scattering angles at $W=1.65 \mathrm{GeV}$ and for different values of $Q^{2}$. Experimental data are from the CLAS collaboration [11]. Solid (dashed) lines are obtained from fit to electroproduction (both photo- and electroproduction) data, whereas dash-dotted lines display the predictions of Kaon-Maid.

This is true for both PS and PV models, although the presently available data cannot resolve the difference between the two models.

In the case of kaon electroproduction, a comparison between unpolarized differential cross sections $\sigma_{\mathrm{U}}$ calculated from the present work and Kaon-Maid models [6] with experimental data is shown in Fig. 2. In this case it is clear that both PS and PV models can nicely reproduce the data and their difference is hardly seen. The same result is also found in case of the polarized structure function $\sigma_{\mathrm{LT}^{\prime}}$ [4]. However, we notice that Kaon-Maid is unable to reproduce the available data in all cases and, in fact, it shows a backward peaking behavior for $\sigma_{\mathrm{U}}$, in contrast to the result of experimental measurement. We suspect that this behavior originates from the large contributions of the $K^{*}$ and $K_{1}$ intermediate states.

\section{III. $K^{0} \Lambda$ PRODUCTION NEAR THRESHOLD}

By using isospin symmetry and appropriate coupling constants obtained from PDG [1], Kaon-Maid [6], as well as Pion-Maid [12], the isobar model developed for the $K^{+} \Lambda$ channel in the previous section can be easily extended to predict observables in the $K^{0} \Lambda$ channel [13]. The $K^{0} \Lambda$ channel is of interest since in the photoproduction case the $K^{0} t$-channel exchange cannot contribute due to the absence of charge and spin degrees of freedom. Thus, the reaction mechanism is somewhat simpler and one can also investigate the effect the 

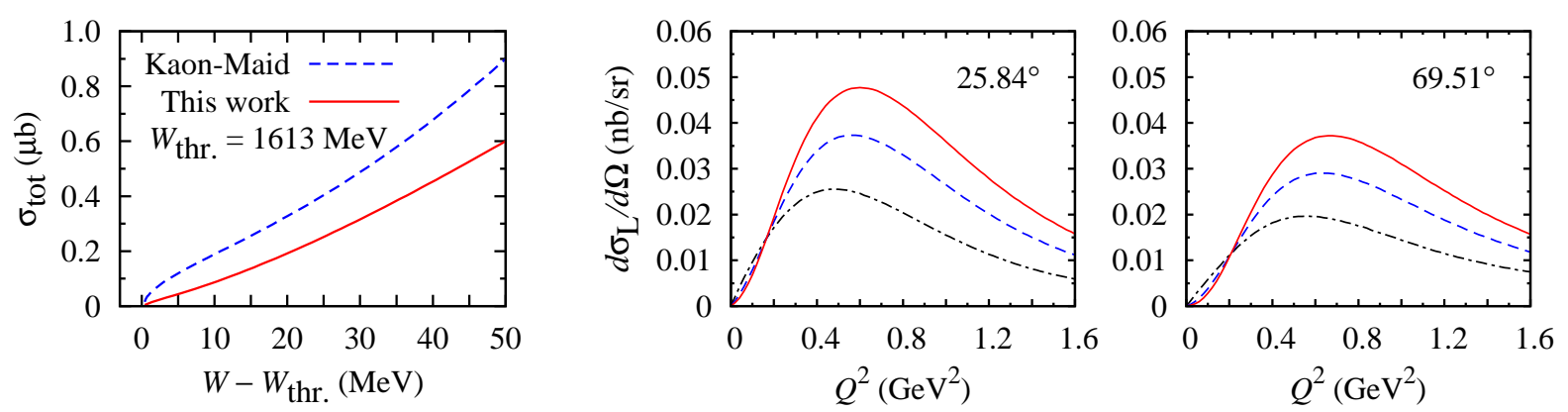

FIG. 3: (Left panel) Comparison between total cross sections of the $\gamma+n \rightarrow K^{0}+\Lambda$ channel predicted by the Kaon-Maid [6] model and the present work. (Middle and right panels) Longitudinal differential cross section of the neutral kaon electroproduction $e+n \rightarrow e^{\prime}+K^{0}+\Lambda$ as a function of the virtual photon momentum squared $Q^{2}$ at $W=1.65 \mathrm{GeV}$ and for different values of the kaon scattering angle. Solid lines show the calculation with a $K^{0}$ form factor obtained in the LCQ model while dashed lines are obtained by using the QMV model. The dash-dotted lines are obtained from a computation with the $K^{0}$ pole excluded.

t-channel by comparing with the $K^{+} \Lambda$ case.

As briefly discussed above, the $K^{0} \Lambda$ electroproduction can be utilized to investigate the effect of $K^{0}$ charge form factor. Compared with other neutral SU(3) pseudoscalar mesons, the neutral kaon has a unique property, i.e. it has an electric or charge form factor. The difference between the strange and non-strange quark masses creates a non-uniform charge distribution in the $K^{0}$. Consequently, although its total charge is zero, the $K^{0}$ has an electric or charge form factor.

The calculated $K^{0} \Lambda$ total cross section is shown in the left panel of Fig. 3, where prediction of the Kaon-Maid is also displayed for comparison. It is apparent that even close to the threshold Kaon-Maid overpredicts our present calculation by a factor of about 30\%. The present calculation has suppressed major uncertainties, such as unknown coupling constants, which could plague the result of Kaon-Maid model. Nevertheless, an experimental check of the $K^{0} \Lambda$ total cross section is still mandatory to help to clarify this situation.

The effects of $K^{0}$ form factors on the longitudinal cross section $\sigma_{\mathrm{L}}$ are shown in the middle and right panels of Fig. 3, where we have employed two relativistic quark models, the light-cone quark (LCQ) model [14] and the quark-meson vertex (QMV) model [15]. It 
is seen from the figure that the effect is sufficiently large for an experimental check and at the forward direction the LCQ form factor raises the cross section up to $50 \%$. Since the resonance contribution is insignificant in this case, this phenomenon clearly originates from the dominant behavior of the background terms. From this figure it is also clear that as the scattering angle increases the effect slightly decreases. Our finding therefore corroborates the finding of Ref. [16], which used the same form factors [14, 15], but different isobar model. Therefore, experimental data with about $10 \%$ uncertainties would be able to pin down the appropriate $K^{0}$ form factor required by the isobar model to describe the $e+n \rightarrow e^{\prime}+K^{0}+\Lambda$ process.

\section{NARROW RESONANCE IN KAON PHOTOPRODUCTION}

Although the existence of the $\Theta^{+}$has become a great puzzle nowadays, interest in the nonstrange partner of $\Theta^{+}$has increased, especially after the finding of a narrow $P_{11}$ nucleon resonance effect in the $\eta$ photoproduction off the neutron [18]. Since the finding of this resonance, a number of theoretical and experimental studies has been devoted for $\eta$ photoproduction off the neutron.

The $\chi$ QSM model predicts that the $P_{11}$ resonance with $J^{P}=1 / 2+$ decays mostly to $\eta N$ channels, whereas the branching ratios to the $\pi N$ and $K \Lambda$ channels are predicted to be

comparable [3]. In the $\pi N$ sector there is only one notable study of this resonance [17]. In this study the narrow $P_{11}$ mass is obtained from $\pi N$ data by using a modified partial wave analysis (PWA), since the standard PWA analysis can miss narrow resonances with $\Gamma<30$ $\mathrm{MeV}$. The changes in the total $\chi^{2}$ were scanned in the range of resonance mass between 1620 to $1760 \mathrm{MeV}$ after the inclusion of this resonance in the $P_{11}$ partial wave. A clear effect was observed at $1680 \mathrm{MeV}$ and a weaker one was detected at $1730 \mathrm{MeV}$. The same result was always obtained although the total width was varied between 0.1 and $10 \mathrm{MeV}$ and branching ratio was also varied between 0.1 and 0.4 .

To our knowledge there has been no attempt to study this resonance by utilizing kaon photoproduction, although kaon photoproduction could offer a new arena for investigating this problem due to the explicit presence of strangeness in the final state. In view of this we decide to scan the changes in the total $\chi^{2}$ after including a $P_{11}$ narrow resonance with the variation of the resonance mass, width, and $K \Lambda$ branching ratio. Such a procedure is 

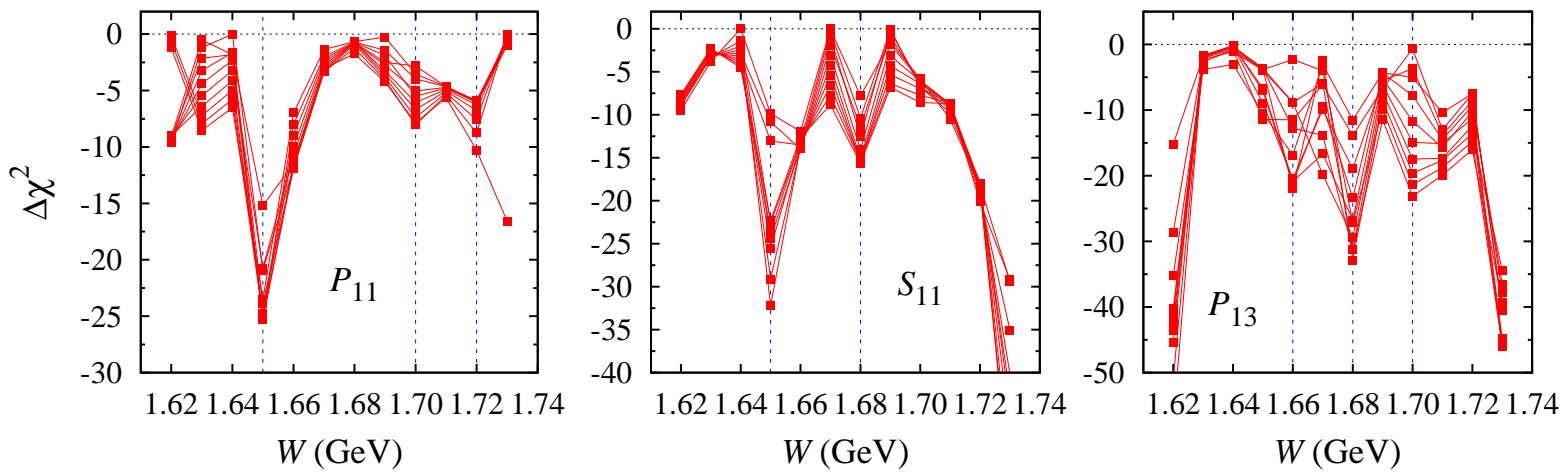

FIG. 4: Change of the $\chi^{2}$ in the fit of kaon photoproduction data due to the inclusion of the $P_{11}$ (left panel), $S_{11}$ (middle panel), and $P_{13}$ (right panel) resonances with masses scanned from 1620 to $1730 \mathrm{MeV}$ (step $10 \mathrm{MeV}$ ) and $\Gamma_{\text {tot. }}$ taken from 1 to $10 \mathrm{MeV}$ (step $1 \mathrm{MeV}$ ) for the $K \Lambda$ branching ratio 0.2 . The vertical lines indicate the possible resonance masses.

apparently suitable for kaon photoproduction, since the cross sections are relatively much smaller than in the case of $\pi N$ or $\eta n$, whereas the experimental error bars are in general relatively larger. As we can see in the next section, it is difficult to observe a clear structure in the cross sections at the energy of interest.

Since we shall scan the resonance mass up to $1730 \mathrm{MeV}$, we need to extend our model explained in the previous sections up to this energy regime. This has been performed with the addition of the $D_{15}(1675), F_{15}(1680), D_{13}(1700), P_{11}(1710)$, and $P_{13}(1720)$ resonance states [21].

The left panel of Fig. 4 displays the result of $\chi^{2}$ changes $\left(\Delta \chi^{2}\right)$ after the inclusion of an extra $P_{11}$ resonance with the mass scanned from 1620 to $1730 \mathrm{MeV}$, where the total width $\Gamma_{\text {tot. }}$ is varied from 1 to $10 \mathrm{MeV}$ with $1 \mathrm{MeV}$ step. We have also investigated the variation of the $K \Lambda$ branching ratio $\Gamma_{K \Lambda}$ and found that the best $\chi^{2}$ would be obtained if we used $\Gamma_{K \Lambda}=0.2$ and $\Gamma_{\text {tot. }}=5 \mathrm{MeV}$. In the left panel of Fig. 4 we can see that three minima appear at $m_{N^{*}}=1650,1700$, and $1720 \mathrm{MeV}$. Nevertheless, the minimum $\Delta \chi^{2}$ at $m_{N^{*}}=1650 \mathrm{MeV}$ seems to be the most convincing one.

The middle panel of Fig. 4 exhibits the changes of the $\chi^{2}$ if we replace the $P_{11}$ narrow resonance with an $S_{11}\left(J^{p}=1 / 2^{-}\right)$resonance. Obviously the same minimum at $m_{N^{*}}=1650$ $\mathrm{MeV}$ is retained, but a new one clearly appears at $m_{N^{*}}=1680 \mathrm{MeV}$. The appearance of the minimum $\Delta \chi^{2}$ at $m_{N^{*}}=1650 \mathrm{MeV}$ in the left and middle panels of Fig. 4 indicates that a 
real structure really exists at this energy point, although it is hardly seen in experimental data. However, the fact that both $S_{11}$ and $P_{11}$ could generate this minimum means that a $J^{p}=1 / 2^{-}$narrow resonance is also possible in the kaon photoproduction process.

Finally, the right panel of Fig. 4 displays the changes of the $\chi^{2}$ if we include a $P_{13}$ $\left(J^{p}=3 / 2^{+}\right)$resonance instead of an $S_{11}$ or a $P_{11}$ state. Surprisingly, the minimum at 1650 $\mathrm{MeV}$ almost vanishes and a clear minimum at $1680 \mathrm{MeV}$, as in the case of the $S_{11}$, appears. Besides that, we also observe two weaker minima at 1660 and $1700 \mathrm{MeV}$. However, the minimum at $1680 \mathrm{MeV}$ is interesting in this case, since the possibility that the structure found in the $\eta$ photoproduction off a neutron can be explained by a $P_{13}$ resonance has been discussed in Ref. [20]. In fact, the most convincing result with the smallest $\chi^{2}$ would be obtained if one used a $P_{13}(1685)$ state instead of a $P_{11}$ [20]. Unfortunately, there is a number of meson photoproduction thresholds around $1685 \mathrm{MeV}$. Therefore, unless we could suppress the threshold effects at this energy point, further discussion of the $P_{13}(1685)$ would be meaningless at this stage.

It is obviously important to know which data are really responsible for the minimum at $1650 \mathrm{MeV}$. For this purpose, we have scrutinized contributions of individual data to the $\chi^{2}$ in our fits and found that this minimum originates mostly from the $\Lambda$ recoil polarization data as displayed in Fig. 5. From this figure we can see that there exists a dip at $W \approx 1650 \mathrm{MeV}$ in the whole angular distribution of data. It is also apparent that both $P_{11}$ and $S_{11}$ states can nicely reproduce the dip. Therefore, it seems to us that the recoil polarization is not the suitable observable to distinguish the possible states at $1650 \mathrm{MeV}$. Nevertheless, more precise recoil polarization data are still urgently required in order to remove uncertainties in the position of the dip.

We have also investigated total and differential cross sections, as well as other polarization observables such as target and photon asymmetries, double polarization observables $O_{x^{\prime}}, O_{z^{\prime}}$, $C_{x}$, and $C_{z}$. It is found that total cross section and double polarization observables $O_{x^{\prime}}, O_{z^{\prime}}$ are the appropriate observables to determine the quantum number and parity of this narrow resonance [21]. 


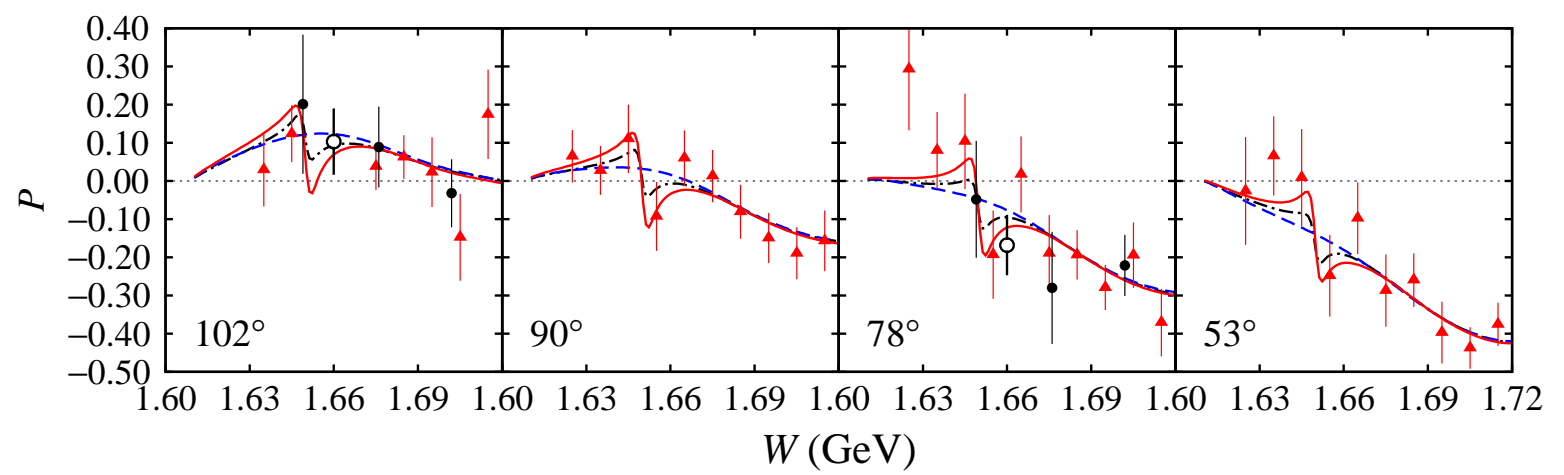

FIG. 5: $\Lambda$ recoil polarization without the inclusion of the narrow resonance (dashed lines), with the inclusion of an $S_{11}$ resonance (dash-dotted lines) and a $P_{11}$ resonance (solid lines) for different values of kaon scattering angles. Experimental data are from SAPHIR [8] (open circles), GRAAL 10] (solid circles), and CLAS [19] (solid triangles) collaborations.

\section{SUMMARY AND CONCLUSION}

We have investigated the electromagnetic production of $K^{+} \Lambda$ and $K^{0} \Lambda$ by means of isobar models. It is found that by using very limited free parameters we can explain all $K^{+} \Lambda$ experimental data nicely. By using isospin symmetry and appropriate parameters obtained from PDG and Maid we predict the $K^{0} \Lambda$ observables and demonstrate the possibility of investigating the $K^{0}$ form factor in the neutral kaon electroproduction.

We have also observed the existence of the $J^{p}=1 / 2^{+}$narrow resonance, the nonstrange member of antidecuplet baryons predicted by the $\chi$ QSM, in kaon photoproduction off a proton. We found the most promising mass and width of this resonance are $1650 \mathrm{MeV}$ and $5 \mathrm{MeV}$, respectively. This finding is observed to be model independent and could be distinguished from the $J^{p}=1 / 2^{-}$and $3 / 2^{+}$resonances, provided that more precise kaon photoproduction data were available. Furthermore, our conclusion does not change with the variation of the total width and $K \Lambda$ branching ratio of the resonance. Although the mass of the resonance obtained in our calculation $(1650 \mathrm{MeV})$ is slightly different from those obtained from the $\pi N$ and $\eta N$ reactions, such a mass corroborates the calculation utilizing the Gell-Mann-Okubo rule without mixing between the lower-lying nucleonlike octet with the antidecuplet. 


\section{Acknowledgment}

The author thanks Igor I. Strakovsky for fruitful discussions on the search of narrow nucleon resonances. Supports from the University of Indonesia and the Competence Grant of the Indonesian Ministry of National Education are gratefully acknowledged.

[1] K. Nakamura et al., J. Phys. G 37, 075021 (2010).

[2] T. Mart and A. Sulaksono, Phys. Rev. C 74, 055203 (2006).

[3] D. Diakonov, V. Petrov, and M. Polyakov, Z. Phys. A 359, 305 (1997).

[4] T. Mart, Phys. Rev. C 82, 025209 (2010).

[5] R. A. Adelseck and B. Saghai, Phys. Rev. C 42, 108 (1990).

[6] T. Mart, C. Bennhold, H. Haberzettl, and L. Tiator, Kaon-Maid. The interactive program is available at http://www.kph.uni-mainz.de/MAID/kaon/kaonmaid.html. The published versions are available in: T. Mart and C. Bennhold, Phys. Rev. C 61, 012201 (1999); T. Mart, Phys. Rev. C 62, 038201 (2000); C. Bennhold, H. Haberzettl and T. Mart, arXiv:nucl-th/9909022.

[7] S. Steininger and U. G. Meissner, Phys. Lett. B 391, 446 (1997).

[8] K. H. Glander et al., Eur. Phys. J. A 19, 251 (2004).

[9] R. Bradford et al., Phys. Rev. C 73, 035202 (2006).

[10] A. Lleres et al., Eur. Phys. J. A 39, 146 (2009).

[11] P. Ambrozewicz et al., Phys. Rev. C 75, 045203 (2007).

[12] D. Drechsel, S. S. Kamalov and L. Tiator, Eur. Phys. J. A 34, 69 (2007).

[13] T. Mart, Photo- and electroproduction of the $K^{0} \Lambda$ near threshold and effects of the $K^{0}$ electromagnetic form factor, arXiv:1104.2393 [nucl-th], to appear in Phys. Rev. C.

[14] C. Bennhold, H. Ito, and T. Mart, Proceedings of the rth International Conference on the Structure of Baryons, Santa Fe, New Mexico, 1995, p.323.

[15] W. W. Buck, R. Williams, and H. Ito, Phys. Lett. B 351, 24 (1995); H. Ito and F. Gross, Phys. Rev. Lett. 71, 2555 (1993).

[16] T. Mart and C. Bennhold, Nucl. Phys. A 639, 237 (1998).

[17] R. A. Arndt, Ya. I. Azimov, M. V. Polyakov, I. I. Strakovsky, and R. L. Workman, Phys. Rev. 
C 69, 035208 (2004).

[18] V. Kuznetsov et al., Phys. Lett. B 647, 23 (2007).

[19] M. E. McCracken et al., Phys. Rev. C 81, 025201 (2010).

[20] V. Kuznetsov et al., Acta Phys. Polon. B 39, 1949 (2008).

[21] T. Mart, Evidence for the $J^{p}=1 / 2^{+}$narrow state at $1650 \mathrm{MeV}$ in the photoproduction of $K \Lambda$, arXiv:1104.2389 [hep-ph], to appear in Phys. Rev. D. 